\title{
Development and validation of the Maudsley Modified Patient Health Questionnaire (MM-PHQ-9)
}

Phillippa Harrison, Syndi Walton, Diede Fennema, Suqian Duan, Tanja Jaeckle, Kimberley Goldsmith, Ewan Carr, Mark Ashworth, Allan. H. Young and Roland Zahn

\section{Background}

The Patient Health Questionnaire-9 (PHQ-9) is a widely used measure of depression in primary care. It was, however, originally designed as a diagnostic screening tool, and not for measuring change in response to antidepressant treatment. Although the Quick Inventory of Depressive Symptomology (QIDS-SR-16) has been extensively validated for outcome measurement, it is poorly adopted in UK primary care, and, although free for clinicians, has licensing restrictions for healthcare organisation use.

\section{Aims}

We aimed to develop a modified version of the PHQ-9, the Maudsley Modified PHQ-9 (MM-PHQ-9), for tracking symptom changes in primary care. We tested the measure's validity, reliability and factor structure.

\section{Method}

A sample of 121 participants was recruited across three studies, and comprised 78 participants with major depressive disorder and 43 controls. MM-PHQ-9 scores were compared with the QIDS-SR-16 and Clinical Global Impressions improvement scale, for concurrent validity. Internal consistency of the scale was assessed, and principal component analysis was conducted to determine the items' factor structure.

\section{Results}

The MM-PHQ-9 demonstrated good concurrent validity with the QIDS-SR-16, and excellent internal consistency. Sensitivity to change over a 14-week period was $d=0.41$ compared with $d=$ 0.61 on the QIDS-SR-16. Concurrent validity between the paper and mobile app versions of the MM-PHQ-9 was $r=0.67$

\section{Conclusions}

These results indicate that the MM-PHQ-9 is a valid and reliable measure of depressive symptoms in paper and mobile app format, although further validation is required. The measure was sensitive to change, demonstrating suitability for use in routine outcome assessment.

\section{Keywords}

Depression; measurement; digital health; antidepressants; primary care.

\section{Copyright and usage}

(C) The Author(s), 2021. Published by Cambridge University Press on behalf of the Royal College of Psychiatrists. This is an Open Access article, distributed under the terms of the creative commons Attribution licence (http://creativecommons.org/ licenses/by/4.0/), which permits unrestricted re-use, distribution, and reproduction in any medium, provided the original work is properly cited.
Measurement of depressive symptoms is important for determining change over time, such as when assessing response to treatment. The late 20th century brought a rapid increase in the number of rating scales for depression, both clinician- and self-rated. ${ }^{1} \mathrm{~A}$ commonly used clinician-rated scale in depression treatment research is the Hamilton Rating Scale for Depression $\left(\mathrm{HRSD}^{2,3}\right)$, and a commonly used self-rated scale is the Beck Depression Inventory-II, ${ }^{4}$ although it is now unaffordable in some routine clinical settings. The advantages and disadvantages of clinician- versus self-rated scales have been heavily debated, with some clear evidence for discrepancies in scoring between clinicians and patients. ${ }^{5}$ Clinicianrated scales are often hailed as the gold standard of rating scales, but there are many advantages to self-rated scales. One compelling advantage of self-rated measures of depression is their efficiency, especially for use over repeated assessments, requiring less clinician time and, hence, being more cost-effective. The Patient Health Questionnaire-9 (PHQ- $9^{6}$ ) has demonstrated high validity as a self-reported outcome scale in primary care, and is widely used because of its lack of licensing restrictions and brevity. However, the PHQ-9 has some limitations relating to its intervals, specificity of symptoms for depression and predictive ability of symptoms.

The PHQ-9 was devised as a diagnostic screening tool using DSM-IV criteria. ${ }^{7}$ In line with DSM-IV criteria for depression diagnoses, the PHQ-9 assesses depressive symptoms over the past 2 weeks. Yet, rating scales with 1-week intervals are more sensitive to change, making them better suited to treatment or research settings. ${ }^{8}$ Furthermore, when asked to reflect over a longer period, many patients find it hard to accurately remember their symptoms, which can create unreliable data. ${ }^{9}$

Furthermore, the PHQ-9, like the DSM-IV/5, collapses 'feeling down and depressed' and 'feeling hopeless' into one item. Although this may be acceptable for diagnostic purposes, it is problematic for measuring severity. Hopelessness and depressed mood have been found to load onto separate factors in major depressive disorder (MDD), demonstrating the importance of hopelessness in patients who are suicidal, ${ }^{10}$ whereas depressed mood is present across the whole range of depression severity. Furthermore, hopelessness is a key symptom in attributional models of depression where it plays a distinct role from depressed mood. ${ }^{11}$

Previous research has shown somatic symptoms to be poor predictors of remission following antidepressant treatment. ${ }^{12}$ In the Sequenced Treatment Alternatives to Relieve Depression trial, MDD remission was predicted by early improvement in mainly non-somatic symptoms, such as sad mood, for participants taking citalopram. ${ }^{12}$ Research has shown somatic symptoms to vary over time with no intervention, creating an unstable factor structure, whereas non-somatic symptoms, such as depressed mood, remain fairly constant. ${ }^{13}$ Furthermore, somatic symptoms were not predictive of future symptoms, whereas non-somatic symptoms were capable of predicting both somatic and non-somatic future symptoms, hence being more suitable for tracking treatment change. ${ }^{13}$ Additionally, somatic symptoms such as appetite and sleep changes can be a result of medication side-effects rather than antidepressant effects. 
Psychomotor activity is included in the PHQ-9 as a depressive symptom, but has not been found to be a consistent symptom of $\mathrm{MDD}$, and was shown to be sensitive to selective serotonin reuptake inhibitor (SSRI)-induced change only in severe depression. ${ }^{14} \mathrm{~A}$ further study found that Surinam Dutch men were more likely to score lower on psychomotor activity than other Dutch men of the same depression severity, ${ }^{15}$ indicating that different cultural backgrounds can influence likelihood to endorse this item. Huang et al also reported a cultural bias when testing Chinese Americans, who were more than twice as likely to endorse psychomotor activity changes as other cultural groups. ${ }^{16}$ Therefore, differences in PHQ-9 scoring by different cultural groups cannot be fully attributed to differences in depression. Furthermore, patients often overlook the criterion that psychomotor activity should be observed by others to be scored as 'present', resulting in an exaggerated PHQ-9 score.

Another weakness is that the PHQ-9 includes the item 'feeling bad about yourself or that you are a failure, or have let yourself or your family down', which encompasses both self-worth and guilt despite clear evidence that low self-worth is a more consistent symptom than guilt. ${ }^{17-19}$ Therefore, the separation of low selfworth and guilt could improve the scale's sensitivity to change in patients experiencing self-blame.

Finally, the PHQ-9 includes no anxiety-related symptoms following the original separation of the Primary Care Evaluation of Mental Disorders (PRIME-MD) into the PHQ-9 for depressive symptoms and the Generalised Anxiety Disorder Questionnaire (GAD-7) for anxiety symptoms. However, the DSM-5 has now incorporated anxiety with the anxious distress specifier for MDD because of its prognostic implications. ${ }^{7}$ Furthermore, there is evidence that psychological aspects of anxiety are good measures of SSRI response in severe and non-severe MDD. ${ }^{14}$

Hence, despite being a useful outcome measure, there are some weaknesses of the PHQ-9 that hinder its accuracy for measuring change in symptoms over time. It is important to recognise that the PHQ-9 is widely used as a severity measure rather than a diagnostic measure as originally developed, so the timescales and symptoms presented should be tailored to accurately measure the changes in depressive symptoms.

\section{Aims}

In this paper, we developed the Maudsley Modified PHQ-9 (MM-PHQ-9) to address the described weaknesses of the PHQ-9 as a measure of change, and present initial data on its validity and reliability. The long-term goal is to provide a freely available tool for tracking antidepressant response in primary care settings. Our specific aims and hypotheses were as follows:

(a) To determine the concurrent validity of the MM-PHQ-9 with pre-existing self-report measures of depressive symptoms (Quick Inventory of Depressive Symptomology (QIDS-SR$16^{20}$ ) at baseline. We hypothesised that the MM-PHQ-9 would correlate with the QIDS-SR-16 as a measure of the common construct of depressive severity. However, higher concurrent validity with the Very Quick Inventory of Depressive Symptomatology (VQIDS-SR5 ${ }^{21}$ ) was hypothesised than with the QIDS-SR-16, as the VQIDS-SR5 excludes somatic items.

(b) To determine the concurrent validity of baseline MM-PHQ-9 when presented in a mobile app compared with a paper/ online format. We hypothesised that MM-PHQ-9 scores would be correlated when presented in both formats.

(c) To determine the sensitivity, specificity, and positive and negative predictive values of baseline MM-PHQ-9. We hypothesised that the MM-PHQ-9 would have high specificity and sensitivity, similar to the original PHQ-9. (d) To determine the sensitivity to change from baseline to followup in depressive symptoms of the MM-PHQ-9 by comparing it with standard measures that can detect change in symptoms: the self-rated Clinical Global Impression Improvement Scale (CGI-Improvement ${ }^{22}$ ) and change in QIDS-SR-16 scores. We hypothesised that the MM-PHQ-9 would be comparable to the CGI-Improvement and QIDS-SR-16 in its ability to detect changes in symptoms.

(e) To test the inter-item correlation of baseline MM-PHQ-9 to evaluate its reliability as an assessment of depression. MMPHQ-9 items were hypothesised to correlate with one another, as all reflecting the common construct of depression severity.

(f) To investigate the underlying factor structure of the MMPHQ-9 scale, using principal component analysis on baseline scores. We hypothesised that the additional items added will load onto a common depression factor with the existing depression items.

\section{Method}

\section{Design}

Secondary data analysis was conducted on data from the Antidepressant Advisor Study (ADeSS), Neurofeedback in Depression (NeuroMooD)and Sadness is Good ( $\mathrm{SiG}$ ) studies (see Table 1). The ADeSS research portfolio comprised three studies on MDD. ADeSS study 1 was a feasibility trial of a decision-support tool to assist general practitioners with antidepressant prescribing for treatment-resistant MDD. ${ }^{23} \mathrm{ADeSS}$ study 2 was an online recruitment and survey study to investigate combinations of patient factors associated with response to treatments for depression. Participants followed a similar study process to ADeSS study 1 , without the intervention. ADeSS study 3 aimed to provide the proof of concept for using functional magnetic resonance imaging (fMRI) biomarkers to prospectively predict which patients would not benefit from standard SSRI treatment, and comparing fMRI measures with participants without depression. ${ }^{23}$ For the purpose of this analysis, participants from ADeSS studies 1 and 2 were included in the MDD sample, and controls from ADeSS study 3 were included in the control sample. The NeuroMooD trial examined a novel real-time fMRI neurofeedback method in MDD, and also included a case-control crosssectional study recruiting healthy controls. ${ }^{24}$ Both MDD and control samples were included in this analysis. The SiG study aimed to use a method of emotion reappraisal to train healthy participants with a tendency for self-blame to convert their self-blame to sadness, as a more adaptive emotion.

ADeSS study 1, ADeSS study 2 and NeuroMooD participants formed the clinical sample for comparing the MM-PHQ-9 with the QIDS-SR-16 for aim (a), and ADeSS study 3 and NeuroMooD controls formed the control sample. ADeSS studies 1 and 2 formed the clinical sample for comparing the MM-PHQ-9 with the VQIDS-SR5. For aim (b), a subsample of participants from ADeSS studies 1 and 2 completed a mobile app version of the MM-PHQ-9, which was compared with the paper version. For aim (c), all participants' MM-PHQ-9 scores were assessed for sensitivity and specificity. For aim (d), change in MM-PHQ-9 score was compared with CGI-Improvement and QIDS-SR-16 change scores in a subsample of participants from ADeSS studies 1 and 2 and all NeuroMooD study participants. For aim (e), MM-PHQ-9 item scores were analysed for clinical sample inter-item correlation for ADeSS study 1, ADeSS study 2 and NeuroMooD study participants, and scores of ADeSS study 3 controls and NeuroMooD study controls were analysed for control sample reliability. For aim (f), principal component analysis was conducted on MM-PHQ-9 item scores of participants from ADeSS studies 1 and 2. 
Table 1 Descriptive statistics of gender, age and years in education for the clinical and control samples

$\begin{array}{lcccc}\text { Study } & n & \text { Gender, \% female } & \text { Age, years, mean (s.d.) } & \text { Years in education, mean (s.d.) } \\ \text { Clinical } & & & & \\ \quad \text { ADeSS study } 1 & 22 & 81.8 & 51.45(15.12) & 13.91(2.83) \\ \text { ADeSS study } & 51 & 98 & 29.19(9.86) & 15.76(3.44) \\ \quad \text { NeuroMooD } & 5 & 100 & 36.60(6.84) & 17.00(3.08) \\ \text { Control } & 20 & - & - & - \\ \quad \text { SiG } & 5 & 80 & 35.60(14.78) & 17.80(2.59) \\ \text { ADeSS study } 3 & 18 & 83.3 & 42.00(16.16) & 16.61(2.64) \\ \quad \text { NeuroMooD } & \text { No demographic data was available for the SiG study. ADeSS, Antidepressant Advisor Study; NeuroMooD, Neurofeedback in Depression trial; Sig, Sadness is Good study. }\end{array}$

\section{Participants}

Participants from the three studies who had completed the MMPHQ-9 were included in our analysis (ADeSS: $n=73$ MDD and $n=5$ non-MDD control participants; SiG: $n=20$ healthy controls; NeuroMooD: $n=5 \mathrm{MDD}$ and 18 healthy control participants), resulting in a total sample of 121 individuals, with $78 \mathrm{MDD}$ and 43 non-MDD control participants. We had missing demographic data for the $20 \mathrm{SiG}$ participants. The remaining sample of 101 participants was $91.1 \%$ female (7.9\% male, $1 \%$ non-binary), with a mean age of 37.24 (s.d. 15.35) and mean years of education of 15.67 (s.d. 3.26). All participants completed baseline assessments.

Inclusion and exclusion criteria varied for each study and are provided in more detail in the relevant study publications. For the purpose of the current analysis, common inclusion criteria across studies for the MDD sample were as follows: age $\geq 18$ years; proficient in English; and at least moderately severe major depressive syndrome on the PHQ-9 (score $\geq 15$; ADeSS studies 1 and 2) or a diagnosis of recurrent MDD according to the DSM- $5^{7}$ (NeuroMooD). Exclusion criteria for the MDD sample were as follows: unstable medical condition, neurological condition, history of manic/hypomanic episodes, history of schizophreniform symptoms or schizophrenia and current/recent drug misuse.

For the non-MDD control sample, common inclusion criteria across studies were age $\geq 18$ years and proficient in English. Exclusion criteria were a diagnosis of MDD or current major depressive syndrome, and bipolar or psychotic disorders.

Key differences in inclusion criteria were that participants from ADeSS studies 1 and 2 were required to be taking an antidepressant at baseline (or had in past 2 months), whereas this was not the case for NeuroMooD study participants. Participants from ADeSS studies 1 and 2 were permitted to undergo psychotherapy during study participation, but this was an exclusion criteria for the NeuroMooD study. Diagnostic procedures are detailed in the Supplementary Methods available at https://doi.org/10.1192/bjo.2021.953.

\section{Measures}

MM-PHQ-9

The MM-PHQ-9 is an adapted version of the PHQ-9, designed to assess depressive symptoms. ${ }^{6}$ Amendments were made to reflect evidence-based knowledge of depressive symptoms: questions 2 and 3 were separated to represent depressed mood and hopelessness separately. Somatic symptoms (sleep, appetite) and the psychomotor item were omitted. Question 5 was added to assess self-blaming emotions, and was validated ${ }^{17}$ to detect self-blaming emotions in $60 \%$ of patients with MDD. This study found guilt to dissociate from low self-worth, and therefore question 6 was simplified. Intervals for symptom assessment were changed from biweekly to weekly. This necessitated changing the wording of the scale anchors to 'some days' instead of 'several days' and 'every day' instead of 'nearly every day'. As with the PHQ-9, the scale comprises 9 items and the total score is derived from summing item scores, with a total range of 0-27.

\section{QIDS-SR-16}

The QIDS-SR-16 is a self-rated measurement of depressive symptom severity. The measure has 16 items that assess a range of depressive symptoms, including mood and somatic-related symptoms. The measure is scored by summing the highest scoring item from items 1-4; item 5; the highest scoring item from items 6-9; items 10,11,12, 13 and 14; and the highest scoring item from items 15 and 16 . It has good internal consistency $(\alpha=0.86)$ and is highly correlated with the HRSD-24 $(r=0.86)$.

\section{VQIDS-SR5}

The VQIDS-SR5 is a shorter, five-item version of the QIDS-SR-16 that measures the core symptoms of depression: sad mood, selfoutlook, involvement, fatigue and psychomotor slowing. These items reflect those included in the HRSD-6, with the removal of anxiety. ${ }^{25}$ The measure has a reduced focus on somatic symptoms compared with the QIDS-SR-16, and so was a useful comparison in our study against the MM-PHQ-9. The scale has good internal consistency $(\alpha=0.67-0.81)$, and strong concurrent validity with the QIDS-SR-16 $(r=0.90)$.

\section{CGI-Improvement}

The CGI-Improvement (self-rated) is a self-rated measurement scale of change in depression over time, based on a combination of symptoms and functioning level. There are seven ordinal categories, from one (very much improved) to seven (very much worse).

\section{Interventions}

ADeSS study 1 participants had their antidepressant treatment reviewed by their general practitioner within 2 weeks of baseline, and potentially changed to another antidepressant or higher dose, continuing to be changed an unlimited number of times over the 14-week study duration. Follow-up occurred 15-18 weeks after baseline. ADeSS study 2 participants received no intervention and were followed up 15-18 weeks after baseline. NeuroMooD study participants received three neurofeedback intervention sessions over 5 weeks, and were required to remain on the same antidepressant and dose. Follow-up occurred up to 52 days after baseline. No control participants from ADeSS study 3, the NeuroMooD study or $\mathrm{SiG}$ study received any interventions or were followed up on.

\section{Procedure}

The authors assert that all procedures contributing to this work comply with the ethical standards of the relevant national and institutional committees on human experimentation and with the Helsinki Declaration of 1975, as revised in 2008. All procedures involving human participants were approved by the Camberwell \& St Giles NHS Research Ethics Committee (ADeSS study reference number 17/LO/2074; NeuroMooD study reference number 15/LO/ 0577) and Psychiatry, Nursing and Midwifery Research Ethics 
Subcommittee at King's College London ( $\mathrm{SiG}$ study reference number HR-17/18-6151). Written informed consent was obtained from all participants for the studies and for their data to be used for future research. All participants completed a paper or online version of the MM-PHQ-9 at baseline, in addition to other measures, as part of their study participation. A subsample of participants with MDD in ADeSS studies 1 and 2 completed a mobile app version (MooDoC version 1.67.0 for Android, Alloc Modulo Ltd, London, UK) of the MM-PHQ-9 (https://play.google.com/ store/apps/details?id=com.allocmodulo\&hl=en_GB\&gl=US) within approximately 2 weeks of baseline. Participants with MDD completed paper or online follow-up assessments. The ADeSS studies were registered with Clinicaltrials.gov, under registration numbers NCT03628027 (study 1) and NCT04342299 (study 3); the NeuroMooD study was registered with the ISRCTN Registry, under registration number ISRCTN10526888. ADeSS study 2 and $\mathrm{SiG}$ studies were not pre-registered.

\section{Analysis}

Data analysis was conducted with SPSS for Windows version 26 $\left(\mathrm{IBM}^{26}\right)$. Because not all studies administered all of the measures of interest, there were varying sample sizes for different analyses. The distribution of the data was assessed with the Shapiro-Wilk test of normality, and non-parametric adaptations were applied to satisfy the assumptions of a normal distribution.

The analysis proceeded in six stages. First, we aimed to determine the concurrent validity of the MM-PHQ-9 at baseline, with the QIDSSR-16, ${ }^{20}$ and the VQIDS-SR5, ${ }^{21}$ by conducting correlations between the MM-PHQ-9 and each scale. Second, we aimed to determine the concurrent validity of the MM-PHQ-9 at baseline when presented in a mobile app compared with an online and paper format, by conducting correlations between the baseline MM-PHQ-9 and the initial mobile app MM-PHQ-9. Third, we aimed to determine the sensitivity, specificity positive and negative predictive values of the MMPHQ-9 by calculating the proportion of participants from the MDD and control samples with scores $>9$ versus scores $\leq 9$. This was based on the PHQ- 9 cut-off criteria of a score of $>9$ being categorised as moderate depressive symptoms. Fourth, we aimed to determine the sensitivity to change in depressive symptoms of the MMPHQ-9 by comparing it with the CGI-Improvement and change in QIDS-SR-16 scores, by conducting paired sample $t$-tests between MM-PHQ-9 baseline and follow-up scores, and QIDS-SR-16 scores. Cohen's $d$ effect sizes were calculated and compared for each measure, using the formula: $d=$ mean change score/s.d. change score. A correlation was conducted between change on the MM-PHQ-9 from baseline to follow-up and CGI-Improvement ratings. Fifth, we aimed to test the reliability of the MM-PHQ-9 at baseline, to assess the presence and the severity of depression, by conducting a test of internal consistency of the MM-PHQ-9 items and deriving Cronbach's alpha, and we assessed the strength of interitem correlations. Finally, we aimed to use principal component analysis to investigate the underlying factor structure of the MM-PHQ-9, including the new items. Because of correlated factors, an Oblimin rotation with Kaiser normalisation was applied to simplify the factor structure. Factors with Eigenvalues above 1 were retained as true factors. A cut-off point of $\geq 0.7$ was applied to item loadings, to be recognised as loading onto a factor. ${ }^{27}$

\section{Results}

\section{Available data}

Forty-eight participants from the MDD sample had available follow-up data that was collected in the required time period; follow-up data was not collected for the control sample. Thirtyfour participants from ADeSS studies 1 and 2 provided MMPHQ-9 data via a mobile app. Demographic data was available for 101 participants (Table 1). The MDD group in ADeSS study 1 had a significantly higher mean age (mean 51.45 years, s.d. 15.12 years) than the MDD groups in ADeSS study 2 (mean 29.19 years, s.d. 9.86 years) and the NeuroMooD study (mean 36.60 years, s.d. 6.84 years $)(F(2,72)=28.16, P<0.001)$. A post hoc Bonferroni test showed this difference to be significant between ADeSS study 1 and ADeSS study $2(t(21)=22.27, P<0.001)$ and the NeuroMooD study $(t(4)=14.86, P=0.034)$.

\section{MM-PHQ-9 and QIDS-SR-16 scores}

The mean baseline MM-PHQ-9 score for the MDD sample was 17.23 (see Table 2), which is typically considered as a moderately severe symptom level on the standard PHQ-9. The mean baseline QIDS-SR-16 score was also 17.23. In comparison, the mean baseline MM-PHQ-9 total for the control sample was 3.67, which is usually considered as no/minimal symptoms on the standard PHQ-9. The mean baseline QIDS-SR-16 score for the control sample was 3.09.

At follow-up, the mean MM-PHQ-9 score for the MDD sample was 14.40 , which falls into the moderate symptom category on the PHQ-9. This produced a mean decrease in symptoms of $12 \%$ (s.d. 39.51 , range from $90 \%$ increase in symptoms to $95 \%$ decrease). The mean QIDS-SR-16 follow-up score was 13.89, which produced a mean decrease in symptoms of $18 \%$ (s.d. 30.01, range from $38 \%$ increase to $100 \%$ decrease).

\section{Distribution of MM-PHQ-9 and QIDS-SR-16 scores and concurrent validity}

The distribution of MDD and control MM-PHQ-9 total scores combined was examined with a Shapiro-Wilk test of normality, and showed a significantly non-normal distribution (ShapiroWilk $(101)=0.92, \quad P<0.001)$. Similarly, a Shapiro-Wilk test revealed that the QIDS-SR-16 total score was not normally distributed (Shapiro-Wilk $(101)=0.88, P<0.001)$. For the MDD sample, the distribution of the MM-PHQ-9 total scores were normally distributed (Shapiro-Wilk $(78)=0.93, P=0.096$ ), as were the QIDSSR-16 total scores (Shapiro-Wilk $(78)=0.97, P=0.113$ ).

For the control sample, the distribution of the MM-PHQ-9 total scores (Shapiro-Wilk $(23)=0.77, P<0.001$ ) and QIDS-SR-16 total scores (Shapiro-Wilk $(23)=0.89, P=0.016)$ were significantly nonnormally distributed (see Table 3 for all concurrent validity statistics).

\section{Relationship between MM-PHQ-9 and QIDS-SR-16 scores}

A Spearman's correlation was conducted on the relationship between the MM-PHQ-9 and QIDS-SR-16 total scores at baseline. The analysis showed there to be high concurrent validity between the MMPHQ-9 and QIDS-SR-16 in the total sample. A Pearson's correlation was conducted on the MDD sample only, and showed there to be moderate concurrent validity between the MM-PHQ-9 and QIDS-

Table 2 MM-PHQ-9 and QIDS-SR-16 total score mean, s.d. and range

\begin{tabular}{|c|c|c|c|c|c|c|c|c|}
\hline & \multirow[b]{2}{*}{$n$} & \multicolumn{3}{|c|}{ MM-PHQ-9 } & \multirow[b]{2}{*}{$n$} & \multicolumn{3}{|c|}{ QIDS-SR-16 } \\
\hline & & Mean & s.d. & Range & & Mean & s.d. & Range \\
\hline MDD baseline & 78 & 17.23 & 4.49 & $7-26$ & 78 & 17.23 & 3.46 & $7-24$ \\
\hline MDD follow-up & 48 & 14.40 & 6.96 & $1-26$ & 47 & 13.89 & 5.78 & $0-24$ \\
\hline Control baseline & 43 & 3.67 & 3.43 & $0-9$ & 23 & 3.09 & 1.41 & $0-5$ \\
\hline
\end{tabular}


Table 3 Concurrent validity of measures with the MM-PHQ-9

\begin{tabular}{|c|c|c|c|c|}
\hline & $n$ & $r$ & d.f. & $P$-value \\
\hline \multicolumn{5}{|c|}{ QIDS-SR-16 } \\
\hline Total & 101 & 0.83 & 101 & $<0.01$ \\
\hline MDD & 78 & 0.65 & 78 & $<0.01$ \\
\hline Control & 23 & 0.67 & 23 & $<0.001$ \\
\hline \multicolumn{5}{|c|}{ VQIDS-SR5 } \\
\hline MDD & 73 & 0.63 & 73 & $<0.001$ \\
\hline \multicolumn{5}{|c|}{ Mobile app MM-PHQ-9 } \\
\hline MDD & 34 & 0.67 & 32 & $<0.001$ \\
\hline \multicolumn{5}{|c|}{$\begin{array}{l}\text { MM-PHQ-9, Maudsley-modified Patient Health Questionnaire-9; QIDS-SR-16, Quick } \\
\text { Inventory for Depressive symptomatology Self-rated } 16 \text { item version; MDD, major } \\
\text { depressive disorder; VQIDS-SR5, Very Quick Inventory of Depressive Symptomatology - } \\
\text { Self-Report. }\end{array}$} \\
\hline
\end{tabular}

SR-16 total scores. This relationship was of similar strength for controls on whom a Spearman's correlation was conducted.

\section{Relationship between MM-PHQ-9 and VQIDS-SR5 scores}

For a subsample of the MDD sample with available data, the VQIDS-SR5 total score was calculated. A Spearman's correlation showed the concurrent validity between VQIDS-SR5 and MMPHQ-9 total scores to be similar to that of the full QIDS-SR-16.

\section{Mobile app MM-PHQ-9 score}

There was a moderate correlation between participants' baseline MM-PHQ-9 total score and their mobile app MM-PHQ-9 total score, indicating good concurrent validity between the two formats.

\section{Sensitivity and specificity}

The sensitivity of the MM-PHQ-9 cut-off score of $\geq 10$ was $98.7 \%$ (true positives: $n=77$ out of 78 participants with MDD; Supplementary Fig. 1 and Table 4), with a specificity of $100.0 \%$ (true negatives: $n=43$ out of 43 control participants), positive predictive value of $100.0 \%$ (true positives: $n=77$ out of the sum of True and False Positives $(n=77))$ and negative predictive value of $97.7 \%$ (true negatives: $n=43$ out of the sum of true and false negatives $(n=44))$. As the prevalence of MDD in our sample was much higher than one would expect in a naturalistic sample, we also calculated predictive values adjusted for $15 \%$ MDD prevalence, to illustrate how these results could translate to future samples (positive predictive value of $100 \%$ and negative predictive value of $99.77 \%)$.

\section{Sensitivity to change}

One participant with QIDS-SR-16 data was excluded from this analysis because of a missing item on the QIDS-SR-16 at follow-up. For those in the MDD sample with follow-up data available (MM-PHQ$9 n=48$; QIDS-SR-16 $n=47$ ), paired $t$-tests were conducted between baseline and follow-up MM-PHQ-9 and QIDS-SR-16 scores. Change in MM-PHQ-9 scores was significant $(t(47)=2.82$, $P=0.007$ ), with a small effect size (Cohen's $d=0.41$ ). Change in QIDS-SR-16 scores was also significant $(t(46)=4.20, P<0.001)$, but with a medium effect size (Cohen's $d=0.61$ ).

There was also a moderate significant relationship between MMPHQ-9 change and self-rated CGI-Improvement at follow-up $(r(45)$ $=-0.64, P<0.001)$. The correlation was negative because a lower CGI-Improvement score indicates larger improvement; however, it shows that more improvement on the MM-PHQ-9 was associated with more improvement on the CGI-Improvement. For reference, the correlation between the QIDS-SR-16 follow-up score and CGIImprovement score was also moderate $(r(44)=-0.56, P<0.001)$.

\section{Internal consistency}

Internal consistency of the MM-PHQ-9 was assessed for the total sample, and participants with MDD and controls separately at baseline. Cronbach's alpha for the internal consistency of the MMPHQ-9 for the total sample was excellent $(\alpha(101)=0.93)$. Interitem correlations were at a moderate level, which indicated that items were measuring the same construct but were not too high to be redundant on an individual item basis. Cronbach's alpha was lower for the MDD sample than for the total sample, but was still good $(r(78)=0.76)$. Inter-item correlations were lower than for the total sample and did not exceed 0.6. Some items appeared to have no relationship, such as item 1 ('Little interest or pleasure in doing things') and item 5 ('Worrying that you have done something wrong'), which correlated at $r=-0.01$. Cronbach's alpha in the control sample was good $(\alpha(23)=0.78)$, and similar to that of the MDD sample. There was a similar relationship between items 1 and 5 for controls and the MDD sample, in that the items were unrelated. There was no indication that deletion of any of items would substantially improve the Cronbach's alphas of the scales.

\section{Principal component analysis}

Principal component analysis was conducted on the MM-PHQ-9 item scores provided by 73 participants from ADeSS studies 1 and 2. A Kaiser-Meyer-Olkin test revealed the data to be appropriate for factor analysis (0.91), and a Bartlett's test of sphericity showed adequate homogeneity of variance $\left(\chi^{2}(36)=690.60\right.$, $P<0.001)$. The component matrix is included in Table 4 , and the pattern, structure and component correlation matrices are included in Supplementary Tables 1-3). The component matrix shows all items to load above 0.4 onto factor 1, which had an Eigenvalue of 5.81 and explained $64.6 \%$ of the total variance, indicating a common construct, whereas factor 2 added an additional $11.4 \%$ of explained variance. The pattern and structure matrices (Supplementary Tables 1 and 2) showed that factor 1 was most strongly associated with symptoms that are thought to be an expression of overgeneralised self-blame, ${ }^{28}$ such as hopelessness, worries about one's mistakes and low self-worth. In contrast, factor 2 was inversely related with symptoms and factor 1 (Supplementary Table 3). Interestingly, the strongest negative associations of factor 2 were found with lack of interest/pleasure, energy and concentration.

\section{Discussion}

Our results show that the MM-PHQ-9 exhibited good concurrent validity when compared with standard self-rated measures of depression. The MM-PHQ- 9 is designed to be used repeatedly by individuals to indicate change in symptoms over time; hence, the

\section{Table 4 Principal component matrix loadings of MM-PHQ-9 items}

\begin{tabular}{|c|c|c|}
\hline Component & 1 & 2 \\
\hline Little interest or pleasure & 0.794 & -0.431 \\
\hline Feeling down or depressed & 0.888 & -0.091 \\
\hline Feeling hopeless & 0.868 & 0.225 \\
\hline Feeling tired or having little energy & 0.815 & -0.443 \\
\hline Worrying that you have done something wrong & 0.767 & 0.429 \\
\hline Feeling bad about yourself & 0.837 & 0.287 \\
\hline Trouble concentrating on things & 0.766 & -0.426 \\
\hline Feeling nervous, anxious or on edge & 0.823 & 0.157 \\
\hline $\begin{array}{l}\text { Thoughts that you would be better off dead or hurting } \\
\text { yourself }\end{array}$ & 0.650 & 0.333 \\
\hline
\end{tabular}

Oblimin rotation with Kaiser normalisation was applied. MM-PHQ-9, Maudsley-modified Patient Health Questionnaire-9. 
change in MM-PHQ-9 from baseline to follow-up was compared with a standard measure of change, the CGI-Improvement, which demonstrated a moderate relationship. The measure demonstrated $100 \%$ specificity and positive predictive values, and almost $100 \%$ sensitivity and negative predictive values, when differentiating the MDD and control samples according to a MM-PHQ-9 cut-off score of 10. This indicates the measure's potential to differentiate those with significant symptoms of depression more likely to be associated with MDD from those with mild symptoms observed in the general population, but this result needs to be interpreted with caution as it could have been driven by sample selection biases, and needs to be investigated in a naturalistic sample to probe its generalisability to clinical settings.

Although the QIDS-SR-16 indicated a larger effect size for detecting change in symptoms, the MM-PHQ-9 was able to detect changes at $d=0.41$, which is approaching a moderate effect size. Further validation studies are needed to determine whether sensitivity to change in response to antidepressant treatment is comparable to that of the QIDS-SR-16, which cannot be determined in our sample, which consisted of a mix of different types of treatment and naturalistic outcomes. It will be important to determine whether the MM-PHQ-9's greater reliance on symptom pervasiveness compared with the QIDS-SR-16's focus on severity may make the latter more sensitive to change.

The MM-PHQ-9 had good internal consistency for the MDD sample, indicating that the items can reliably assess depressive symptoms. The newly added items to assess hopelessness, selfblame and anxiety correlated with other items at a level comparable to the original PHQ-9 items, implying that the new items are relevant for depression severity. However, when the sample was split into MDD and healthy control participants, the newly added item 5 ('Worrying that you have done something wrong') correlated negatively with original items 1 ('Little interest or pleasure in doing things') and 4 ('Feeling tired or having little energy'). In contrast, item 8 ('Feeling nervous, anxious or on edge') correlated positively with all original PHQ-9 items, which indicates that anxiety is indeed strongly associated with other core symptoms.

Interestingly, this partial dissociation of self-blame-related and anhedonia-related items is also reflected in the two factors derived from our principal component analysis, which revealed one main factor, interpreted as a common depression factor as it reflects variance common to all items but is most strongly associated with overgeneralised self-blame-related items, and a second factor that is inversely related and reflects interest, pleasure and motivation more specifically. A previous body of research has found evidence for a two-factor structure of PHQ-9 items, namely a somatic and a non-somatic factor. ${ }^{13,29-31}$ As we removed somatic items when creating the MM-PHQ-9 it was expected that no somatic factor would remain, which is in line with our findings.

We also demonstrated that the mobile app version of the MMPHQ-9 had good concurrent validity with the paper/electronic survey version. Digital mental health is rapidly developing, providing opportunities for individuals to independently monitor their symptoms in a quick and easy manner. Hence, it is key for research to compare accuracy of results via smartphone and standard presentation before utilising technology for depression rating scales and for the MM-PHQ-9 to be validated as suitable for further e-health applications.

\section{Limitations and future research}

Despite indications of good concurrent validity and reliability, there are several limitations that must be acknowledged. The research is limited by variable inclusion criteria across the different data-sets. ADeSS study 1 specified a diagnosis of MDD as its inclusion criteria, and the NeuroMooD study specified a diagnosis of recurrent MDD, both according to the DSM-5. However, ADeSS study 2 did not recruit a clinically diagnosed sample, instead requiring a PHQ-9 score of $\geq 15$. Despite the lack of diagnostic inclusion criteria for the total sample limiting the clinical application of the MM-PHQ9 , the range of severity and chronicity present in this study can be beneficial for creating a measure that captures the full spectrum of depressive severity. Measures of depression often have too limited ranges to capture those with both non-severe and severe depression. ${ }^{14}$ Therefore, a universal measure such as the MMPHQ-9, which has been tested on individuals with and without a current diagnosis or not fully remitted, could improve the accuracy of self-reported symptoms without the need for separate assessments for different severities of depression.

Similarly, the ranging study designs meant that the studies were not homogenous in treatment types and durations. Participants from ADeSS studies 1 and 2 were taking antidepressants at baseline (or had in past 2 months), whereas NeuroMooD study participants were mixed in antidepressant use. Participants from ADeSS studies 1 and 2 were permitted to undergo psychotherapy during study participation, but NeuroMooD study participants were not. ADeSS study 1 participants had their antidepressant treatment reviewed within 2 weeks of baseline and potentially changed to another antidepressant or higher dose, whereas study 2 participants were not reviewed, and NeuroMooD study participants were required to remain on the same antidepressant and dose and received neurofeedback. Because of the potentially mixed impact on response of the different treatments, the sensitivity to change findings of $d=$ 0.41 for the MM-PHQ-9 should be viewed tentatively. However, the comparative QIDS-SR-16 sensitivity to change of $d=0.61$ provides some reassurance that the MM-PHQ-9 captured real change in symptoms over time.

Another limitation arising from the various data sources was a small sample size resulting from not all studies administering all measures of interest. This limits the confidence that can be had in some of the findings, particularly for the small sample on whom mobile app delivery of the MM-PHQ-9 was tested. Although there are twice as many women than men with MDD, both our clinical and control samples comprised over $80 \%$ women. Additionally, the result of our factor analysis may have been biased by specific characteristics of our sample, and will require replication in a sample that also includes a broader range of patients with MDD at different stages of recovery. Conversely, many other participant characteristics were widely heterogenous, such as age and depression subtype. Although such heterogeneity is beneficial for capturing a pragmatic sample to whom findings can be generalised, this approach lacks the specificity required to understand differences in response to a new measure by different subgroups. Future research should also probe the influence of item wording, which has been shown to be suboptimal for the standard PHQ-9 from the patient perspective. ${ }^{32}$

The mobile app used for data collection was developed as a pilot study in the ADeSS. Because of app installation issues, initial mobile app scores could have been submitted up to approximately 2 weeks after baseline, which could have reduced the relationship between the scores at the two time points. This is particularly important for ADeSS study 1 data, in which participants may have changed antidepressant within the 2-week period, although the effects of this would likely have a delayed effect. Future research should aim to compare app and paper MM-PHQ-9 scores for the same time point, to provide stronger alternate-form validity. In summary, our data provides preliminary evidence that the MM-PHQ-9 is a valid and reliable outcome measure of core depressive symptoms. The data is freely available for use and further validation in larger samples. 
Phillippa Harrison, Department of Psychological Medicine, Centre for Affective Disorders, Institute of Psychiatry, Psychology \& Neuroscience, King's College London, UK Syndi Walton, Department of Psychological Medicine, Centre for Affective Disorders, Institute of Psychiatry, Psychology \& Neuroscience, King's College London, UK; Diede Fennema, Department of Psychological Medicine, Centre for Affective Disorders, Institute of Psychiatry, Psychology \& Neuroscience, King's College London, UK; Suqian Duan, Department of Psychological Medicine, Centre for Affective Disorders, Institute of Psychiatry, Psychology \& Neuroscience, King's college London, UK: Tanja Jaeckle, Department of Psychological Medicine, Centre for Affective Disorders, Institute of Psychiatry, Psychology \& Neuroscience, King's College London, UK; Kimberley Goldsmith (1D), Department of Biostatistics and Health Informatics, Institute of Psychiatry, Psychology \& Neuroscience, King's College London, UK; Ewan Carr, Department of Biostatistics and Health Informatics, Institute of Psychiatry, Psychology \& Neuroscience, King's College London, UK; Mark Ashworth (iD), School of Population Health and Environmental Sciences, King's College London, UK; Allan. H. Young (D. Department of Psychological Medicine, Centre for Affective Disorders, Institute of Psychiatry, Psychology \& Neuroscience, King's College London, UK; and National Service for Affective Disorders, South London and Maudsley NHS Foundation Trust, UK Roland Zahn (D), Department of Psychological Medicine, Centre for Affective Disorders, Institute of Psychiatry, Psychology \& Neuroscience, King's College London, UK; and National Service for Affective Disorders, South London and Maudsley NHS Foundation Trust, UK

Correspondence: Roland Zahn. Email: roland.zahn@kcl.ac.uk

First received 25 Jan 2021, final revision 17 May 2021, accepted 26 May 2021

\section{Supplementary material}

Supplementary material is available online at https://doi.org/10.1192/bjo.2021.953

\section{Data availability}

The anonymised data that support the findings of this study are available from the corresponding author, R.Z., upon reasonable request.

\section{Acknowledgements}

We thank Jingiing Zhang and Sofia Chanturishvili for their roles in data collection and cleaning and Kevin Kermani Nejad and Dr Maximilian Kerz from Alloc Modulo for developing the mobile app version of the questionnaire. We also thank our research participants.

\section{Author contributions}

P.H. formulated the research question, collected and analysed the data and drafted the manuscript. S.W. and T.J. contributed intellectually to the research question and collected data. D.F. and S.D. collected data and commented significantly on the manuscript. K.G. E.C., M.A. and A.H.Y. commented significantly on the manuscript. R.Z. formulated the research question, guided data analysis and contributed significantly to the manuscript. All authors agree to be accountable for the accuracy and integrity of the research, and have provided intellectual contribution and final approval of the manuscript to be published.

\section{Funding}

This research has received funding from the National Institute for Health Research (NIHR) Research for Patient Benefit (grant reference PB-PG-0416- 20039), the NIHR Biomedical Research Centre at South London and Maudsley NHS Foundation Trust and King's College London, and the Medical Research Council Doctoral Training Partnership (grant reference 2064430). This paper represents independent research part-funded by the NIHR Biomedical Research Centre at South London and Maudsley NHS Foundation Trust and King's College London, and NIHR Applied Research Collaboration South London at King's College Hospital NHS Foundation Trust. The views expressed are those of the author(s) and not necessarily those of the National Health Service, the NIHR or the Department of Health and Social Care. E.C. is fully funded by the NIHR Biomedical Research Centre, and K.G. is part-funded by the $\mathrm{NIHR}$ Biomedical Research Centre and NIHR Applied Research Collaboration South London. Funding was also partly provided by the Rosetrees Trust (grant M816).

\section{Declaration of interest}

Allan Young is a consultant for Johnson \& Johnson and LivaNova; has given paid lectures and sat on advisory boards for the following companies, regarding drugs used in affective and related disorders: AstraZeneca, Eli Lilly, Lundbeck, Sunovion, Servier, LivaNova, Janssen, Allegan, Bionomics and Sumitomo Dainippon Pharma; received honoraria for attending advisory boards and presenting talks at meetings organised by LivaNova; is the Principal Investigator of the following studies: the Restore-Life Vagus Nerve Stimulation registry study funded by LivaNova, 'An Open-label, Long-term, Safety and Efficacy Study of Intranasal Esketamine in Treatment-resistant Depression', 'The Effects of Psilocybin on Cognitive Function in Healthy Participants' and 'The Safety and Efficacy of Psilocybin in Participants with TreatmentResistant Depression'; and received grant funding (past and present) from the following institutions: National Institutes of Mental Health (USA), Canadian Institutes of Health Research, Brain \& Behavior Foundation (USA), Stanley Medical Research Institute (USA), Medical Research Council (UK), Wellcome Trust (UK), Royal College of Physicians (Edinburgh), British Medical Association (UK), Vancouver General Hospital Foundation (Canada), Western Economic Diversification Canada, Depression Research Fund (Canada), Michael Smith Foundation for Health Research (Canada), National Institutes for Health Research (UK) and Janssen (UK). He has no shareholdings in pharmaceutical companies. Roland Zahn is a private psychiatrist service provider at The London Depression Institute, and co-investigator on the LivaNova-funded observational study 'Vagus Nerve Stimulation for Depression'; received honoraria for talks at medical symposia sponsored by Lundbeck and Janssen; has collaborated with EMIS group PLC for this study and advises Depsee Ltd; and is affiliated with the D'Or Institute of Research and Education, Rio de Janeiro, and advises the Scients Institute Palo Alto. Kim Goldsmith reports grants from National Institutes of Health Research (UK), Stroke Association (UK), National Institutes of Health (USA) and Juvenile Diabetes Research Foundation (USA), during the conduct of the study. Ewan Carr reports personal fees from National Institutes of Health Research, during the conduct of the study. The other authors report no competing interests. Roland Zahn and Allan Young are members of the BJPsych Open Editorial Board, and as such, were not involved in decisions regarding this manuscript.

\section{References}

1 Kearns NP, Cruickshank C, McGuigan K, Riley S, Shaw SP, Snaith R. A comparison of depression rating scales. Br J Psychiatry 1982; 141(1): 45-9.

2 Bagby RM, Ryder AG, Schuller DR, Marshall MB. The Hamilton Depression Rating Scale: has the gold standard become a lead weight? Am J Psychiatry 2004; 161(12): 2163-77.

3 Hamilton M. The Hamilton Depression Scale-accelerator or break on antidepressant drug discovery. Psychiatry 1960; 23: 56-62.

4 Beck AT, Ward CH, Mendelson M, Mock J, Erbaugh J. An inventory for measuring depression. Arch Gen Psychiatry 1961; 4(6): 561-71.

5 Cuijpers P, Li J, Hofmann SG, Andersson G. Self-reported versus clinician-rated symptoms of depression as outcome measures in psychotherapy research on depression: a meta-analysis. Clin Psychol Rev 2010; 30(6): 768-78.

6 Kroenke K, Spitzer RL. The PHQ-9: a new depression diagnostic and severity measure. Psychiatr Ann 2002; 32(9): 509-15.

7 American Psychiatric Association. Diagnostic and Statistical Manual of Mental Disorders, Fifth Edition (DSM-5). American Psychiatric Publishing, 2013.

8 Fok CCT, Henry D. Increasing the sensitivity of measures to change. Prev Sci 2015; 16(7): 978-86

9 Thomas DL, Diener E. Memory accuracy in the recall of emotions. J Pers SoC Psychol 1990; 59(2): 291

10 van Ballegooijen W, Eikelenboom M, Fokkema M, Riper $\mathrm{H}$, van Hemert AM, Kerkhof AJ, et al. Comparing factor structures of depressed patients with and without suicidal ideation, a measurement invariance analysis. J Affect Disord 2019: 245: 180-7.

11 Abramson LY, Seligman ME, Teasdale JD. Learned helplessness in humans critique and reformulation. J Abn Psychol 1978; 87(1): 49-74.

12 Sakurai $\mathrm{H}$, Uchida $\mathrm{H}$, Abe T, Nakajima S, Suzuki T, Pollock BG, et al. Trajectories of individual symptoms in remitters versus non-remitters with depression. J Affect Disord 2013; 151(2): 506-13.

13 Krause JS, Reed KS, McArdle JJ. Factor structure and predictive validity of somatic and nonsomatic symptoms from the Patient Health Questionnaire-9: a longitudinal study after spinal cord injury. Arch Phys Med Rehabil 2010; 91(8): 1218-24.

14 Hieronymus F, Lisinski A, Nilsson S, Eriksson E. Influence of baseline severity on the effects of SSRIs in depression: an item-based, patient-level post-hoc analysis. Lancet Psychiatry 2019; 6(9): 745-52.

15 Baas KD, Cramer AO, Koeter MW, Van De Lisdonk EH, Van Weert HC, Schene AH. Measurement invariance with respect to ethnicity of the Patient Health Questionnaire-9 (PHQ-9). J Affect Disord 2011; 129(1-3): 229-35.

16 Huang FY, Chung H, Kroenke K, Delucchi KL, Spitzer RL. Using the Patient Health Questionnaire-9 to measure depression among racially and ethnically diverse primary care patients. J Gen Intern Med 2006; 21(6): 547-2.

17 Zahn R, Lythe KE, Gethin JA, Green S, Deakin JFW, Young AH, et al. The role of self-blame and worthlessness in the psychopathology of major depressive disorder. J Affect Disord 2015; 186: 337-41.

18 Ebert $\mathrm{D}$, Martus $\mathrm{P}$, Lungershausen $\mathrm{E}$. Change in symptomatology of melancholic depression over two decades. Psychopathology 1995; 28(5): 273-80.

19 Prosen M, Clark DC, Harrow M, Fawcett J. Guilt and conscience in major depressive disorders. Am J Psychiatry 1983; 7: 839-44.

20 Rush AJ, Trivedi MH, Ibrahim HM, Carmody TJ, Arnow B, Klein DN, et al. The 16-item Quick Inventory of Depressive Symptomatology (QIDS), Clinician Rating (QIDS-C), and Self-Report (QIDS-SR): a psychometric evaluation in patients with chronic major depression. Biol Psychiatry 2003; 54(5): 573-83.

21 De La Garza N, John Rush A, Grannemann BD, Trivedi MH. Toward a very brie self-report to assess the core symptoms of depression (VQIDS-SR5). Acta Psychiatr Scand 2017; 135(6): 548-53.

22 Busner J, Targum SD, Miller DS. The Clinical Global Impressions scale: errors in understanding and use. Compr Psychiatry 2009; 50(3): 257-62.

23 Harrison $\mathrm{P}$, Carr E, Goldsmith K, Young AH, Ashworth M, Fennema D, et al. Study protocol for the antidepressant advisor (ADeSS): a decision support system for antidepressant treatment for depression in UK primary care: a feasibility study. BMJ Open 2020; 10(5): e035905. 
24 Jaeckle T, Williams SC, Barker GJ, Basilio R, Carr E, Goldsmith K, et al. Self-blaming emotions in major depression: a randomised pilot trial comparing fMRI neurofeedback training with self-guided psychological strategies (NeuroMooD) medRxiv [Preprint] 2019. Available from: https://www.medrxiv.org/content/ 10.1101/19004309v1 [cited 2021].

25 Bech P, Gram L, Dein E, Jacobsen O, Vitger J, Bolwig T. Quantitative rating of depressive states: correlation between clinical assessment, Beck's self-rating scale and Hamilton's objective rating scale. Acta Psychiatr Scand 1975; 51(3): 161-70.

26 IBM Corporation. IBM SPSS Statistics for Windows, Version 26.0. IBM Corporation, 2019 (https://www.ibm.com/uk-en/analytics/spss-statisticssoftware).

27 Stevens J. Applied Multivariate Statistics for the Social Sciences (3rd edn). Lawrence Erlbaum Associates, 1996.

28 Zahn R, Lythe KE, Gethin JA, Green S, Deakin JF, Workman C, et al. Negative emotions towards others are diminished in remitted major depression. Eur Psychiatry 2015; 30(4): 448-53.
29 Elhai JD, Contractor AA, Tamburrino M, Fine TH, Prescott MR, Shirley E, et al The factor structure of major depression symptoms: a test of four competing models using the Patient Health Questionnaire-9. Psychiatry Res 2012; 199 (3): 169-73.

30 Richardson EJ, Richards JS. Factor structure of the PHQ-9 screen for depression across time since injury among persons with spinal cord injury. Rehabil Psychol 2008; 53(2): 243

31 Whisman MA, Perez JE, Ramel W. Factor structure of the Beck Depression Inventory-Second Edition (BDI-II) in a student sample. J Clin Psychol 2000; 56(4): 545-51.

32 Malpass A, Dowrick C, Gilbody S, Robinson J, Wiles N, Duffy L, et al. Usefulness of PHQ-9 in primary care to determine meaningful symptoms of low mood: a qualitative study. Br J Gen Pract 2016; 66(643): e78-84.

AXTRA 